The presence of other bacteria in CIN or in normal cervical tissues lacked significance likely due to sample size, and additional investigation is required.

\section{P1.21 COMPARISON OF SHIPPED VERSUS FRESHLY FROZEN SELF-COLLECTED VAGINAL SAMPLES FOR MICROBIOTA ASSESSMENT}

${ }^{1}$ Brotman R, ${ }^{1} \mathrm{P}$ Gajer, ${ }^{1}$ Holm Jb, ${ }^{1}$ Robinson $\mathrm{Cr},{ }^{2} \mathrm{D}$ Jones, ${ }^{1} \mathrm{~A}$ Chatterjee, ${ }^{1} \mathrm{M}$ Humphrys, ${ }^{3}$ Forney Lj, ${ }^{1} \mathrm{~J}$ Ravel, ${ }^{2}$ Ghanem Kg. 'Institute for Genome Sciences, University of Maryland School of Medicine, Baltimore, USA; ${ }^{2}$ Division of Infectious Diseases, Johns Hopkins School of Medicine, Baltimore, USA; ${ }^{3}$ Department of Biological Sciences, University of Idaho, Moscow, USA

\subsection{6/sextrans-2017-053264.129}

Introduction Studies have confirmed that self-collected and clinician-collected mid-vaginal swabs sample the same microbial diversity. Self-collected samples shipped through the mail for PCR-based STI testing has also been validated. We sought to determine if the microbiota of shipped vaginal samples are concordant with freshly frozen samples.

Methods In January-February 2016, 20 women self-collected six mid-vaginal swabs which were then stored in two different nucleic acid preservatives (3 E-swabs in $2 \mathrm{ml}$ of modified C2 (MO BIO) and 3 in $2 \mathrm{ml}$ of Amies/RNALater). Modified C2 was selected for ease of use in laboratory processing and DNA extraction. For each set of 3 swabs, 2 were immediately frozen $\left(-80^{\circ} \mathrm{C}\right)$ and one was sent at room temperature to the University of Idaho in a FedEx "Clinical Pak" which was then return shipped to Baltimore. Meta-taxonomic analysis was performed by sequencing the V3-V4 regions of the $16 \mathrm{~S}$ rRNA gene. Hierarchical clustering of vaginal microbiota was used to assign community state types (CST) to each sample. Bayesian hierarchical models were applied to perform within-women comparisons of shipped and freshly frozen samples.

Results Average duration of transit for the shipped samples was 8 days (SD: 1.60, range: 6-11). Paired comparison of CSTs between a woman's shipped and freshly frozen samples as well as between C2 and Amies/RNALater revealed no differences (100\% concordance, kappa: 1.0 for both). After correction for multiple testing, no significant differences between phylotype relative and absolute abundances were detected in C2 or Amies/RNALater groups. Similarly, there were no statistically significant differences between total bacterial loads of shipped versus freshly frozen samples in C2 (p-value: 0.47 ) or Amies/RNALater (p-value: 0.21) samples.

Conclusion There were no differences in vaginal microbiota composition and structure between a woman's shipped and freshly frozen vaginal samples stored in Amies/RNAlater or C2. These data enable future studies to allow participants to self-collect and mail vaginal microbiota specimens.

\section{P1.22 VITAMIN D AND THE VAGINAL MICROBIOME: RESULTS FROM A BLINDED, RANDOMISED CONTROLLED TRIAL}

${ }^{1}$ Holm Jb, ${ }^{2} \mathrm{X}$ He, ${ }^{1}$ Brotman Rm, ${ }^{3}$ Turner An. 'Institute for Genome Sciences, University of Maryland School of Medicine, Baltimore, USA; ${ }^{2}$ Department of Epidemiology and Biostatistics, University of Maryland School of Public Health, College Park, USA; ${ }^{3}$ Division of Infectious Diseases, College of Medicine, Ohio State University, Columbus, USA

10.1136/sextrans-2017-053264.130
Introduction A blinded, randomised controlled trial (RCT) previously demonstrated no effect of vitamin D supplementation on recurrence of bacterial vaginosis (BV) diagnosed by Nugent score. Here, we examine archived samples to determine whether vitamin D supplementation affected trial participants' vaginal microbial composition.

Methods Women with symptomatic BV via Amsel criteria were recruited and treated with $500 \mathrm{mg}$ of oral metronidazole at baseline. Participants were randomised to vitamin D supplementation ( 9 doses of 50,000 IU cholecalciferol over 24 weeks) or matching placebo. Vaginal bacterial composition was characterised for 15 women in each treatment arm, using samples collected at baseline, 4 and 24 weeks. We sequenced the V1-V3 region of the $16 \mathrm{~S}$ rRNA gene and taxonomy was assigned by PECAN. Microbiota were clustered into 5 community state types (CSTs) using Bray-Curtis distances and hierarchical clustering with Ward linkage. We assessed serum vitamin D levels using the Liaison $25 \mathrm{OH}$ vitamin D total assay.

Results We observed no significant effect of vitamin D treatment on Lactobacillus dominance over 24 weeks $(p>0.5)$. Additionally, serum vitamin D levels were not associated with CST $(p=0.22)$. Following metronidazole treatment, the Lactobacillus iners-dominated CST (III) was more common at week 4 compared to enrollment in both the placebo and vitamin D groups. Specifically, the relative abundance of L. iners was significantly higher in the placebo arm at weeks $4 \quad(\mathrm{p}<0.001)$ and $24(p=0.04)$ compared to the vitamin D arm.

Conclusion In agreement with the RCT, we observed no association between vitamin $\mathrm{D}$ supplementation and the vaginal microbiota. While many women in both RCT groups tended toward L. iners-dominated microbiotas following metronidazole treatment at week $4, L$. iners was significantly more abundant in the placebo group. Future research may examine if vitamin D plays a role in stimulating non-lactobacilli growth and how supplements, in addition to antibiotics, affect the emergence of robust lactic acid producing lactobacilli.

\section{P1.23 ANTIBIOTIC USAGE AND COMMENSAL PHARYNGEAL NEISSERIA OF MSM IN HANOI, VIETNAM}

${ }^{1}$ Huan Vinh Dong, ${ }^{2}$ Nguyen Thi Hoa, ${ }^{3}$ Nguyen Xuan Binh Minh, ${ }^{3}$ Nguyen Vu Trung, ${ }^{4}$ Folasade May, ${ }^{3}$ Le Minh Giang, ${ }^{5}$ Jeffrey D Klausner. ${ }^{1}$ Charles R. Drew University of Medicine and Science, David Geffen School of Medicine at UCLA, LOS Angeles, USA; ${ }^{2}$ National Hospital of Tropical Diseases, Hanoi - North Vietnam; ${ }^{3}$ Hanoi Medical University, Hanoi - North Vietnam; ${ }^{4}$ David Geffen School of Medicine at UCLA; Los Angeles, USA; ${ }^{5}$ David Geffen School of Medicine at UCLA; Fielding School of Public Health at UCLA, LoS Angeles, USA

\subsection{6/sextrans-2017-053264.131}

Introduction: Neisseria gonorrhoea (NG) is gaining resistance to last line cephalosporins; conferring resistance to almost all antimicrobials used to treat it since the 1930s. NG disproportionately affects men-who-have-sex-with-men (MSM) and sex workers. Neisseria are particularly apt at horizontal gene transmission within the genus. Genetic analysis of resistant NG found fragments from $N$. cinerea and N. perflava, common commensals of the oropharynx. Nearly all global cases of ceftriaxone resistant NG are reported from pharyngeal samples. Self-medication with antibiotics is prevalent in Vietnam and MSM of Hanoi have high rates of STIs.

Methods MSM from Hanoi, Vietnam were surveyed regarding health seeking behaviours, including antibiotic usage. 
Pharyngeal swabs from a subset of participants were collected and assessed for commensal Neisseria prevalence, as well as characterisation of antimicrobial susceptibility to ciprofloxacin, cefixime, ceftriaxone, and cefpodoxime.

Results Thirty-nine MSM were surveyed. Median age was 23, $72.7 \%$ have sex only with men, and $18.2 \%$ have exchanged sex to receive food, money, or work. Any antibiotic use was $82 \%$ in the past 6 months and $33 \%$ in the past 30 days. In the past 6 months, use of cefixime and ceftriaxone was $15 \%$ and $9 \%$, respectively. Ever use of antibiotics without a prescription was 64\%.At least one commensal Neisseria was isolated from every participant, with prevalence of $N$. cinerea (14.3\%), N. flavescens (57.1\%), N. mucosa (28.6\%), N. subflava (50\%). Using CLSI MIC breakpoints for N. gonorrhoea as a proxy, "reduced susceptibility" to ciprofloxacin, cefixime, ceftriaxone, and cefpodoxime was found among 47.1\%, $11.8 \%, 11.8 \%$, and $29.4 \%$ of all isolates, respectively; $52.9 \%$ were "resistant" to ciprofloxacin.

Conclusion MSM of Hanoi were likely to take antibiotics without a prescription. Commensal Neisseria were very prevalent from pharyngeal samples; some may have reduced susceptibility or resistance to common antibiotics. More data is needed to appropriately interpret antimicrobial susceptibility for these commensals.

\section{P1.24 MYCOPLASMA GENITALIUM AND TRICHOMONAS VAGINALIS DETECTION IN A COHORT OF MEN WHO HAVE SEX WITH MEN IN BELGIUM: EVALUATION OF THE DIAGENODE S-DIAMGTV MULTIPLEX KIT}

Irith De Baetselier, Hilde Smet, Bea Vuylsteke, Tania Crucitti. Institute of Tropical Medicine, Antwerp, Belgium

\subsection{6/sextrans-2017-053264.132}

Introduction: Mycoplasma genitalium (MG) and Trichomonas vaginalis (TV) are Sexually Transmitted Infections (STIs) frequently detected in Non-chlamydial Non-gonococcal urethritis, however the prevalence of MG and TV in urine (U), anorectal (A) and pharyngeal (P) samples is unknown among men who have sex with men (MSM) in Belgium. The objective was to evaluate the MG/TV multiplex kit of Diagenode on the three different sample types.

Methods In the context of an ongoing study among MSM, UA-P samples are collected every three months to test for STIs including MG and TV. DNA extraction is performed on the Abbott m2000sp system. The DNA extracts are tested in both an accredited in-house MG pdhD- gene real-time PCR (RTPCR) for MG (target: pdhD gene) and the S-DiaMGTV multiplex kit of Diagenode for MG (target: mgpa and mg219 gene) and TV (target: TV-spec repeat gene). In addition, in case of a positive TV result, the sample is tested by an inhouse TV RT-PCR (TV-spec repeat gene). All amplifications were performed using the Rotor-GeneQ MDx HRM (Qiagen) platform.

Results To date, of the 1779 samples that were tested for MG/TV, 1768 provided a valid result. Only one anorectal sample was positive for TV but this result could not be confirmed by the in-house TV RT-PCR assay. MG was detected by both assays in 64 samples. The S-DiaMGTV assay missed 3/2/1 U-A-P infections and identified additional 2/2/2 U-A-P infections. Both assays were $99.3 \%$ concordant for MG. Up to now an overall prevalence of $4.0 \%$ for $M G$ is found: $4.8 \%(29 / 598) ; 6.4 \%(37 / 578)$ and $0.7 \%$ (4/592) for U-A-P, respectively. Compared to the in-house RT-PCR the SDiaMGTV showed an overall sensitivity/specificity of 91.4\%/ 99.7\%: 89.7\% (CI 95\%: 72.7\%-97.8\%)/99.7\% (CI 95\%: 98.7\%-100\%) for urine; 94.6\% (CI 95\%: 81.8\%-99.3\%)/ 99.6\% (CI 95\%: 98.7\%-100.0\%) for anorectal and $75.0 \%$ (CI 95\%: 19.4\%-99.4\%)/99.7\% (CI 95\%: 98.8\%-100.0\%) for pharyngeal samples.

Conclusion MG but not TV is prevalent in MSM in urine and anorectal samples. The S-DiaMGTV multiplex kit is an appropriate assay to detect MG in urine and anal samples.

\section{P1.25 COLLI-PEE: A NEW DEVICE TO COLLECT FIRST-VOID URINE AT HOME FOR MOLECULAR DETECTION OF STIS. EVALUATION \& ACCEPTABILITY BY MSM IN A PREP STUDY IN BELGIUM}

${ }^{1}$ Irith De Baetselier, ${ }^{2}$ Vanessa Vankerckhoven, ${ }^{2}$ Manon De Koeijer, ${ }^{1}$ Thijs Reyniers, ${ }^{1}$ Hilde Smet, ${ }^{1}$ Bea Vuylsteke, 'Tania Crucitti. 'Institute of Tropical Medicine, Antwerp, Belgium; ${ }^{2}$ Novosanis, Wijnegem, Belgium

\subsection{6/sextrans-2017-053264.133}

Introduction The CE-labelled Colli-Pee device efficiently captures first-void urine without interruption of the urine stream and is suited for postal delivery. We aimed to evaluate the use of Colli-Pee collected urine mailed by regular post for sexually transmitted infection (STI) detection.

Methods A Belgian study cohort of men who have sex with men were asked to collect first-void urine using the Colli-Pee at home the day after the study visit. At the visit, a first-void urine sample was collected in a regular recipient to detect Neisseria gonorrhoeae (NG), Chlamydia trachomatis (CT), Mycoplasma genitalium (MG) and Trichomonas vaginalis (TV) using commercial and in house Nucleic Acid Amplification Techniques (NAATs). The Colli-Pee collector tube was sent back to the laboratory by regular post and the same NAATs were performed. A survey was completed by the participants on the usability and willingness to use the Colli-Pee device.

Results To date, 265 results were taken into account. Using Colli-Pee, one additional CT was detected and one CT was missed $(5 / 5)$ compared to the regular recipient. Two additional NG $(7 / 5)$ and 2 additional MG were detected but one MG was missed using the Colli-Pee (16/15). TV was not detected in any of the urine samples. The Kappa agreement was $79,6 \%, 83 \%$ and $89,7 \%$ for CT, NG and MG, respectively. On average, Colli-Pee samples were taken 1.7 days (0-22 days) after the regular samples and were on route for an average of 4,3 days (0-17 days). $89 \%$ found the Colli-Pee easy to use, but $16 \%$ remarked that the Colli-Pee is not recyclable.

Conclusion The two STIs (CT/MG) that were missed using the Colli-Pee were only weak positive in the regular sample. More STIs were detected in the Colli-Pee samples: 1 CT, $2 \mathrm{NG}$ and $2 \mathrm{MG}$. However, the Colli-Pee samples were collected 1, 2, 3, 8, and unknown days later than the regular urines, we cannot exclude that participants became positive or infected during that time frame. In conclusion, we showed that the Colli-Pee can be used for home collection of firstvoid urine and postal delivery for STI testing. 